\title{
Cell responses and hemocompatibility of $g$-HA/PLA composites
}

\author{
LI Jia ${ }^{1,2}$, ZHENG Wei ${ }^{1}$, ZHENG YuFeng ${ }^{1,3^{*}} \&$ LOU Xia ${ }^{1,4}$ \\ ${ }^{1}$ Center for Biomedical Materials and Engineering, Harbin Engineering University, Harbin 150001, China; \\ ${ }^{2}$ School of Chemistry and Environmental Engineering, Harbin University of Science and Technology, Harbin 150040, China; \\ ${ }^{3}$ Department of Advanced Materials and Nanotechnology, College of Engineering, Peking University, Beijing 100871, China; \\ ${ }^{4}$ Nanochemistry Research Institute, Department of Chemical Engineering, Curtin University of Technology, Bentley WA 9102, Australia
}

Received May 10, 2009; accepted May 9, 2010; published online March 17, 2011

\begin{abstract}
The objective of this study was to investigate the hemocompatibility and cell responses to some novel poly(L-lactide) (PLA) composites containing surface modified hydroxyapatite particles for potential applications as a bone substitute material. The surface of hydroxyapatite (HA) particles was first grafted with L-lactic acid oligomers to form grafted HA ( $g$-HA) particles. The $g$-HA particles were further blended with PLA to prepare $g$-HA/PLA composites. Our previous study has shown significant improvement in tensile properties of these materials due to the enhanced interfacial adhesion between the polymer matrix and HA particles. To further investigate the potential applications of these composites in bone repair and other orthopedic surgeries, a series of in vitro and in vivo experiments were conducted to examine the cell responses and hemocompatibility of the materials. In vitro experiments showed that the $g$-HA/PLA composites were well tolerated by the L-929 cells. Hemolysis of the composites was lower than that of pure PLA. Subcutaneous implantation demonstrated that the $g$-HA/PLA composites were more favorable than the control materials for soft tissue responses. The results suggested that the $g$-HA/PLA composites are promising and safe materials with potential applications in tissue engineering.
\end{abstract}

polylactide, hydroxyapatite, composites, biocompatibility, cytotoxicity

Citation: $\quad$ Li J, Zheng W, Zheng Y F, et al. Cell responses and hemocompatibility of $g$-HA/PLA composites. Sci China Life Sci, 2011, 54: 366-371, doi: $10.1007 / \mathrm{s} 11427-011-4155-0$

Hydroxyapatite (HA) is one of the most important ceramics in clinical applications because of its similarity to bone minerals in both structure and composition and excellent bioactivity and osteoconductivity [1]. However, its brittleness and low mechanical strength limits its applications in load bearing implants. A number of HA/polymer composites have attracted much attention to offset HA mechanical weakness [2,3]. Among them, poly(L-lactide) (PLA) is commonly used as a matrix material in constructing biodegradable composites for bone repairing [4,5].

As filler, however, HA does not strongly adhere to the PLA matrix. This weak adhesion is due to the significant chemical dissimilarity between HA and PLA. The weak

*Corresponding author (email: yfzheng@pku.edu.cn) interfacial layer between the filler and the matrix can be readily destroyed after implantation; therefore, HA particles easily escape from the polymer matrix leading to a rapid decrease in mechanical strength. Improvement of the interfacial adhesion between the HA particles and the PLA matrix has become critical in preparing HA/PLA composites [6].

Recently, we reported a new and simple method for preparing L-lactic acid oligomer grafted HA ( $g$-HA)/PLA composites [7]. Compared to ordinary HA/PLA composites $[8,9]$, the tensile strength and modulus were effectively improved. The $g$-HA particles demonstrated improved adhesion with PLA matrix, and the $g$-HA/PLA composites showed greater enhancement of tensile strength and elastic modulus in comparison with non-modified HA/PLA com- 
posites [7]. We intend to further assess these materials for possible bone repair and clinical applications.

In this work, the in vitro cytotoxicity and in vivo biocompatibility of $g$-HA/PLA composites were investigated. The cytotoxicity of the $g$-HA/PLA composites was investigated using L929 cells as model cells. The blood compatibility was evaluated by a hemolysis test. Histological evaluation of the materials after subcutaneous implantation, up to eight weeks in rabbits, was reported.

\section{Materials and methods}

\subsection{Preparation of $g$-HA/PLA composite rods}

The $g$-HA particles were prepared as described in our previous work [7]. The method for the preparation of $g$-HA is briefly depicted in Figure 1. To HA particles that were uniformly dispersed in tetrahydrofuran, L-lactic acid was added dropwise. After the toluene was added, the mixture was preserved at $85^{\circ} \mathrm{C}$ for $6-8 \mathrm{~h}$. After washing with ethyl acetate, the particles were polymerized with L-lactide at $130^{\circ} \mathrm{C}$ for $24 \mathrm{~h}$ with stannous octoate as a catalyst. The product was suspended in chloroform and separated by centrifugation. They were further washed with excessive chloroform and vacuum-dried yielding L-lactic acid oligomer grafted HA, $g$-HA.

The $g$-HA particles and PLA were placed in a mould (10 $\mathrm{cm} \times 8 \mathrm{~cm} \times 1.1 \mathrm{~cm}$ ) and pressed to make slices under 6.0 $\mathrm{MPa}$ of pressure at $160^{\circ} \mathrm{C}$ for $20 \mathrm{~min}$. Then the slices were cut and shaped into rods with a diameter of $2.5 \mathrm{~mm}$ and length of $1 \mathrm{~cm}$. The rods of neat PLA were made by the same method.
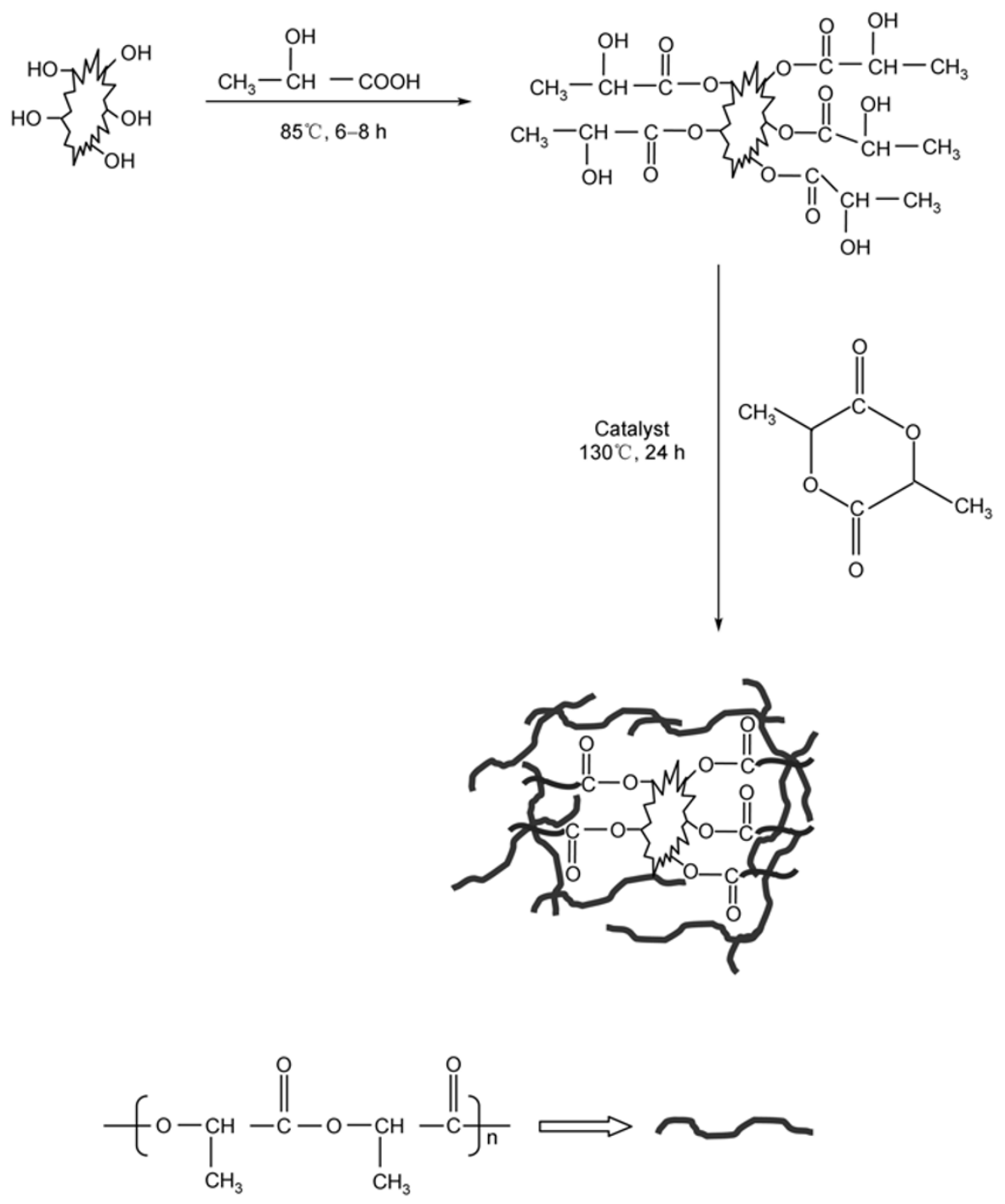

Figure 1 Schematic illustration for the preparation of $g$-HA particles. 


\subsection{Scanning electron microscopy observations}

A scanning electron microscope (SEM; FEI Sirion 200, Philips) was used to observe the morphology of the fractured surface of the composites. A layer of gold was plated uniformly over the fractured surfaces before examination.

\subsection{In vitro cell responses}

Murine fibroblast cells (L-929) were used to examine the cell responses to $g$-HA/PLA composites. L-929 cells were cultured in Dulbecco's modified Eagle's medium (DMEM), $10 \%$ fetal bovine serum (FBS), $100 \mathrm{U} \mathrm{mL}^{-1}$ penicillin and $100 \mu \mathrm{g} \mathrm{mL}{ }^{-1}$ streptomycin at $37^{\circ} \mathrm{C}$ in a humidified atmosphere of $5 \% \mathrm{CO}_{2}$. The tests were carried out by indirect contact.

Extracts were prepared using DMEM serum free medium as the extraction medium. The samples were immersed into DMEM at a ratio of $1.25 \mathrm{~mL} \mathrm{~cm}{ }^{-2}$ (medium/sample) in a humidified atmosphere with $5 \% \mathrm{CO}_{2}$ at $37^{\circ} \mathrm{C}$ for $72 \mathrm{~h}$ according to the International Organization for Standardization (ISO 10993-5:1999 [10] and ISO 10993-12:1996 [11]), then filtered with a $0.22 \mu \mathrm{m}$ sterile membrane. All extracts were used within $24 \mathrm{~h}$. DMEM medium was used as a negative control and DMEM medium with $10 \%$ dimethylsulfoxide (DMSO) was a positive control. Cells were seeded into 96-well plates at a density of $5 \times 10^{3}$ cells/well and incubated for $24 \mathrm{~h}$ at $37^{\circ} \mathrm{C}$ to allow attachment. The culture medium was removed and $100 \mu \mathrm{L}$ extract was added to the wells. After incubating the cells in a humidified atmosphere with $5 \% \mathrm{CO}_{2}$ at $37^{\circ} \mathrm{C}$ for 1,2 and $3 \mathrm{~d}$, the 96 -well plates were observed under an optical microscope. After this, $10 \mu \mathrm{L}$ sterile MTT (3-(4,5-dimethylthiazol-2-yl)-2,5-diphenyltetrazolium bromide) was added to each well. The samples were incubated with MTT for $4 \mathrm{~h}$ at $37^{\circ} \mathrm{C}$, then $100 \mu \mathrm{L}$ of formazan solution was added, followed by incubation overnight. The absorbance of the samples at $570 \mathrm{~nm}$ was measured with an Elx-800 spectrophotometer (bio-Tek instruments).

\subsection{Statistical analysis}

Cell culture experiments were performed at least three times. All the data collected throughout the study were expressed as mean \pm SD. Experimental data were analyzed using Microsoft Excel software. The statistical significance was evaluated by a Student's $t$-test. A $P$-value less than 0.05 was considered statistically significant.

\subsection{Hemolysis tests}

Healthy New Zealand white rabbit blood containing potassium oxalate $\left(1.0 \mathrm{mg} \mathrm{mL}{ }^{-1}\right)$ was taken and diluted with normal saline (4:5 ratio by volume). Specimens of approximately $0.5 \mathrm{~g}(30 \mathrm{~mm} \times 8 \mathrm{~mm} \times 1 \mathrm{~mm})$ were dipped into a standard tube containing $10 \mathrm{~mL}$ of normal saline that was previously incubated at $37^{\circ} \mathrm{C}$ for $30 \mathrm{~min}$. Then $0.2 \mathrm{~mL}$ of diluted blood was added to this standard tube and the mixtures incubated for $60 \mathrm{~min}$ at $37^{\circ} \mathrm{C}$. A normal saline solution was used as a negative control and distilled water as a positive control. After incubation, samples were centrifuged for $5 \mathrm{~min}$. The supernatant was removed and transferred to a cuvette for spectroscopic analysis at $545 \mathrm{~nm}$. The hemolysis was calculated using an ultraviolet spectrophotometer (721; Huichuang Instrument Co., Ltd., China). The hemolysis value was calculated based on the average of five replicates:

$$
\begin{aligned}
\text { Hemolysis }= & \frac{A(\text { test })-A(\text { negative control })}{A(\text { positive control })-A(\text { negative control })} \\
& \times 100 \%
\end{aligned}
$$

\subsection{In vivo implantation}

The $5 \mathrm{wt} \% \mathrm{~g}$-HA/PLA composite and PLA rods were rinsed with $70 \%$ ethanol three times, followed by three washes with phosphate-buffered solution (PBS) and dried in a sterilized hood prior to the implantation.

A total of 18 New Zealand white rabbits weighing between 2.5 and $3.5 \mathrm{~kg}$ were used for in vivo implantation of the composite rods. The rabbits were anesthetized by an intravenous injection of Nembutal $\left(30 \mathrm{mg} \mathrm{kg}^{-1}\right)$. The operations were performed under standard aseptic conditions.

After four $1.5 \mathrm{~cm}$ incisions were made in the shaved skin of each rabbit's dorsum, four subcutaneous pockets were created bluntly. A rod sample, either pure PLA or $5 \mathrm{wt} \%$ $g$-HA/PLA composite was inserted in each of the pockets (Figure 2A) and the skin then sutured. Following all surgical procedures, the rabbits were kept in cages and maintained on a regular laboratory diet. The rabbits were sacrificed by Nembutal overdose at each of the follow-up intervals which included one, four and eight weeks post-implantation. Tissue surrounding the implanted rod (within $5 \mathrm{~mm}$ ) was retrieved for histological examination (Figure 2B-D).

\subsection{Histological examination}

Retrieved specimens were fixed in $10 \%(\mathrm{v} / \mathrm{v})$ buffered formalin, dehydrated in a graded series of ethanol, and embedded in paraffin. The tissue blocks were then stained with hematoxylin and eosin (H\&E) for observing the nucleus and cytoplasm.

\section{Results and discussion}

\subsection{SEM observations}

The SEM images of the fractured surface of $5 \mathrm{wt} \%$ HA/PLA and $5 \mathrm{wt} \% \mathrm{~g}$-HA/PLA composite are shown in Figure 3. It indicates that $g$-HA particles have a more uniform distribu- 

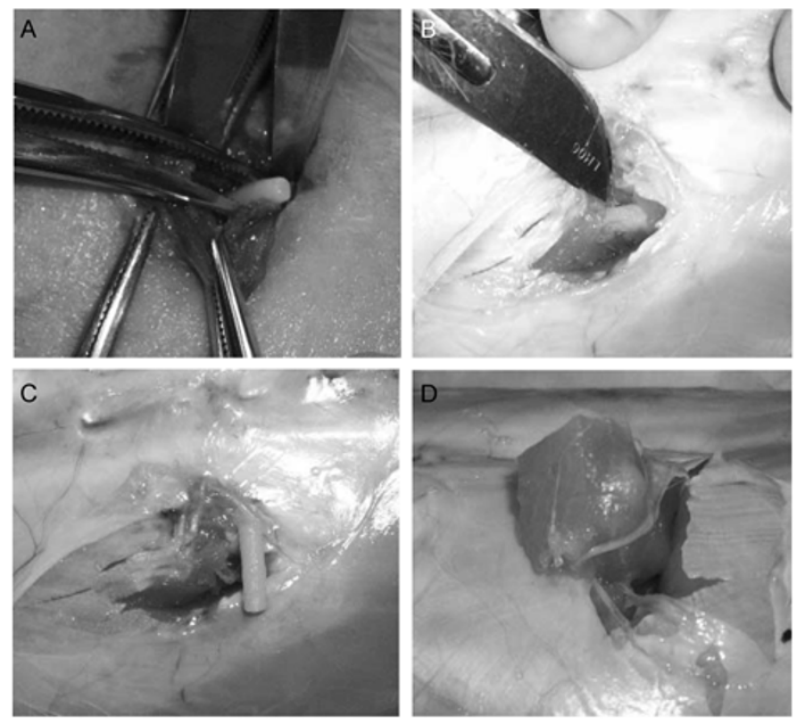

Figure 2 Implantation of $g$-HA/PLA composite rods. A, The composite rod was implanted in a subcutaneous pocket. B, An intraoperative view on the subcutaneously implanted composite rod at eight weeks which could be retrieved easily. $\mathrm{C}$, The explanted composite rod at eight weeks which maintained its original dimension and volume. D, The tissue surrounding the composite rod at eight weeks post-implantation.
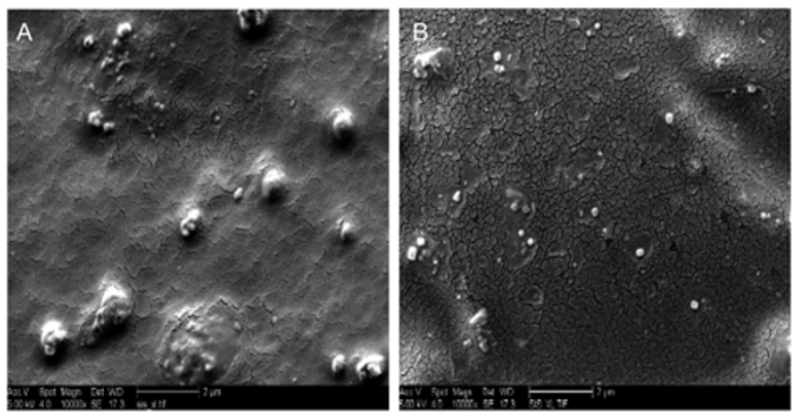

Figure 3 SEM observation of the surface morphologies of $5 \mathrm{wt} \%$ HA/PLA (A) and $5 \mathrm{wt} \% \mathrm{~g}$-HA/PLA composite (B).

tion with few agglomerates when compared with the HA particles.

\subsection{In vitro cell responses of the $g$-HA/PLA composites}

Figure 4 shows the viability of the L-929 murine fibroblast cells expressed as a percentage of the viability of cells cultured in the negative control following culture in pure PLA and $g$-HA composite extraction medium at days 1, 2 and 3 . Although there was a slight decrease in cell viability in both PLA and $g$-HA/PLA extracts in comparison to the negative control, there was no significant difference in cell responses between the PLA and $g$-HA/PLA composite. While the cell viability in PLA was $89.1 \%-98.6 \%$, in $g$-HA/PLA it was about $90.8 \%-97.0 \%$. This indicated that the surface modified $g$-HA/PLA materials can facilitate cell migration and attachment onto the material surfaces as well as PLA. The results also indicated that the composites were not toxic to the cells.

Figure 5 demonstrates the morphologies of L-929 cells cultured in the control samples, and the extracts of PLA and $g$-HA/PLA composites after a 3-d incubation. Healthy morphology of cells with a flattened spindle shape was demonstrated in PLA, $g$-HA/PLA composites and the negative control samples (Figure 5A-E). An abundant growth of cells was observed on the negative control sample (Figure $5 \mathrm{~A}$ ) as well as on the composites (Figure 5C-E). Cell proliferation was limited to PLA (Figure 5B) and suppressed by the toxic substrate in the positive control (Figure 4F). Both the MTT and optical microscopic observation indicated that the $g$-HA/PLA composites can be well tolerated by L-929 cells.

\subsection{Hemocompatibility of $g$-HA/PLA composites}

Figure 6 shows the hemolysis of pure PLA and $g$-HA/PLA

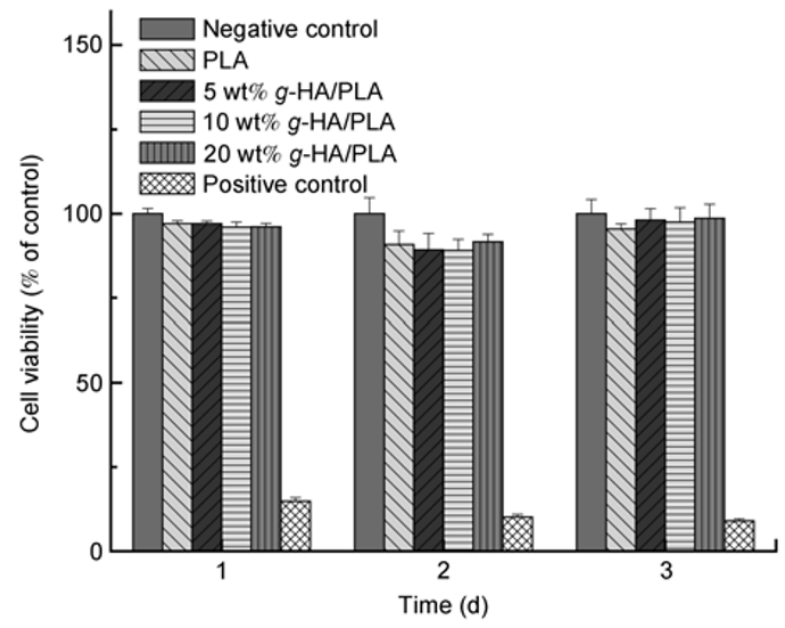

Figure 4 Cell viability expressed as percentage of viable cells in the control after 1, 2 and $3 \mathrm{~d}$ in culture with extraction medium containing pure PLA and $g$-HA/PLA composites.
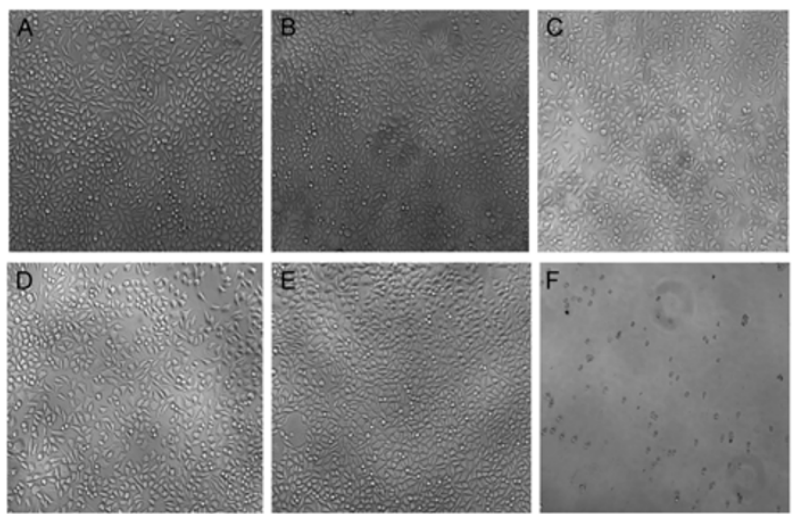

Figure 5 Optical micrographs of L-929 cells that were cultured as a negative control (A), with PLA (B), with 5 wt\% $g$-HA/PLA (C), 10 wt\% $g$-HA/PLA (D), $20 \mathrm{wt} \% g$-HA/PLA (E), and the positive control for $3 \mathrm{~d}$ (F). 


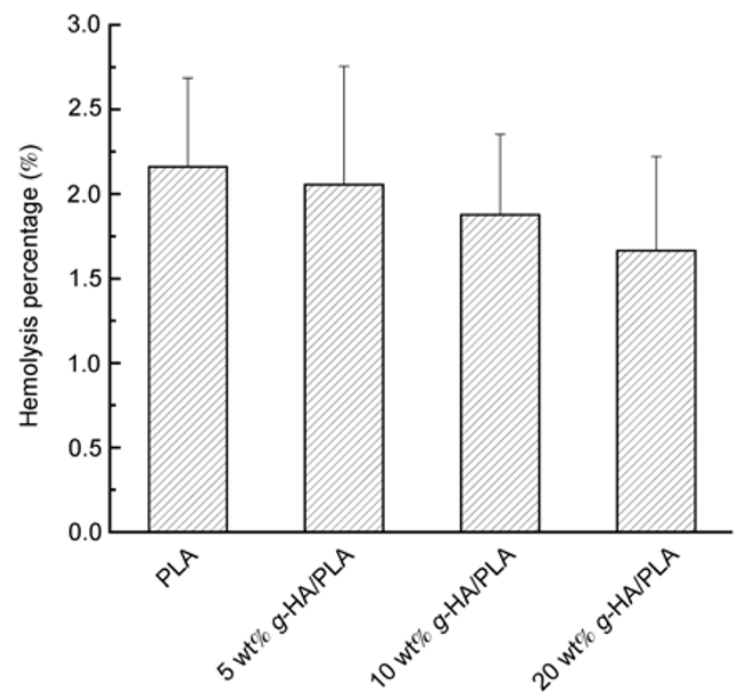

Figure 6 Percent hemolysis of pure PLA and $g$-HA/PLA composites.

composites. In comparison with pure PLA, there is a decrease in hemolysis values in all composite materials. With an increase in $g$-HA content in the composite materials from 5 $\mathrm{wt} \%$ to $10 \mathrm{wt} \%$ and $20 \mathrm{wt} \%$, the hemolysis of the composites decreased from $2.05 \%$ to $1.87 \%$ and $1.66 \%$. The results indicated good hemocompatibility of $g$-HA, and the addition of $g$-HA particles had not deteriorated the biocompatibility of the matrix PLA. Moreover, the hemolysis of pure PLA and $g$-HA/PLA composites was both lower than $5 \%$.

\subsection{In vivo studies of the composites}

In vivo studies indicated that the volume of the rod sample at eight weeks post-implantation was well maintained with a diameter of $2.5 \mathrm{~mm}$, as originally fabricated (Figure 2C). The histological examination results (Figure 7A and $\mathrm{B}$ ) revealed that the fibrous tissues around PLA and the $5 \mathrm{wt} \%$ $g$-HA/PLA composite denaturalized and the structures of fibrous tissue layer could not be distinctly observed after one week. There were many inflammatory cells, such as lymphocytes, neutrophils or macrophages between the fibrous tissue layers. Substances like fibrin exuded from the inflammatory regions. The evident early post operation inflammation was induced by the injured muscle fibers due to the surgical procedure of implantation. The inflammatory cells observed at this stage disappeared four weeks after implantation (Figure 7C and D). Therefore, it was reasonable to conclude that inflammation could be attributed to the injury caused by the implantation procedure.

At four weeks post-implantation, the structures of fibrous tissue around PLA and the $5 \mathrm{wt} \% \mathrm{~g}$-HA/PLA composite were both distinct and arranged regularly. The neutrophils and macrophages could not be observed and few lymphocytes were seen between the fibrous tissues. Compared with the implantations at one week, the inflammatory reaction was obviously weaker. At eight weeks post-implantation, there were no inflammatory cells in the tissue around the $g$-HA/PLA composite (Figure 7E and F). A fibrous tissue layer could be observed with evenly distributed fibrocytes and collagen fibers. In the structure of the fibrous tissue, there were no distinct differences between 4 and 8 weeks post-implantation.

There were no significant differences in soft tissue response to the various materials when implanted subcutaneously (Figure 7A-F), which confirms that the PLA and
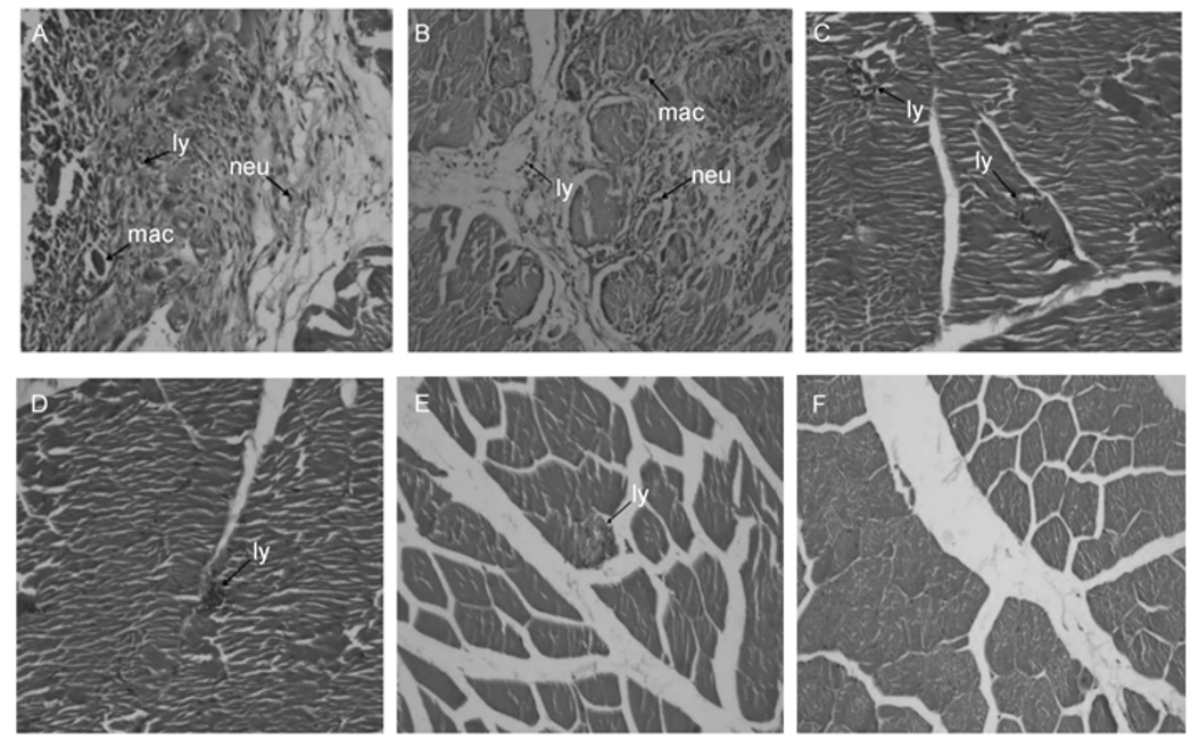

Figure 7 Representative histological sections of the tissues surrounding the rods implanted subcutaneously. A, PLA after one week implantation. B, 5 wt $\%$ $g$-HA/PLA, one week after implantation. C, PLA four weeks after implantation. D, 5 wt\% g-HA/PLA at four weeks post-implantation. E, PLA at eight weeks post-implantation. F, 5 wt $\%$ g-HA/PLA eight weeks after implantation. Images presented are at 20× magnification. ly, lymphocytes; mac, macrophages; neu, neutrophils. 
$g$-HA/PLA composite do not differ in their in vivo biocompatibility when implanted ectopically. Histologically, neither macrophages nor inflammatory giant cells were seen after eight weeks, indicating that $g$-HA/PLA composites are biocompatible and induced no tissue reactions within the experimental period.

\section{Conclusion}

This study investigated the cytotoxicity, hemocompatibility and the inflammatory effect of $g$-HA/PLA materials. The results indicate that the $g$-HA/PLA composites are not toxic to cells and the $g$-HA particles with good hemocompatibility do not deteriorate the biocompatibility of the matrix PLA. Histological examination of the composite materials up to eight weeks after subcutaneous implantation demonstrated that no inflammation was induced by the materials, indicating a good biocompatibility of the $g$-HA/PLA composites. Although further studies on long-term degradation of these materials are needed, the results obtained from the current study are encouraging for clinical usage in various applications.

This work was supported by the Research Fund for the Doctoral Program of Higher Education (Grant No. 20060217012).
1 Viswanath B, Ravishankar N. Controlled synthesis of plate-shaped hydroxyapatite and implications for the morphology of the apatite phase in bone. Biomaterials, 2008, 29: 4855-4863

2 Wen J, Li Y, Zuo Y, et al. Preparation and characterization of nano-hydroxypatite/silicone rubber composite. Mater Lett, 2008, 62: 3307-3309

3 Tang C Y, Chen D Z, Yue T M, et al. Water absorption and solubility of PHBHV/HA nanocomposites. Compos Sci Technol, 2008, 68: 1927-1934

4 Nejati E, Mirzadeh H, Zandi M. Synthesis and characterization of nano-hydroxyapatite rods/poly(l-lactide acid) composite scaffolds for bone tissue engineering. Compos Part A, 2008, 39: 1589-1596

5 Zheng $\mathrm{X}$, Zhou S, Li X, et al. Shape memory properties of poly(D,L-lactide)/hydroxyapatite composites. Biomaterials, 2006, 27: 4288-4295

6 Luong N D, Moon I S, Lee D S, et al. Surface modification of poly(Llactide) electrospun fibers with nanocrystal hydroxyapatite for engineered scaffold applications. Mater Sci Eng C, 2008, 28: 1242-1249

7 Li J, Lu X L, Zheng Y F. Effect of surface modified hydroxyapatite on the tensile property improvement of HA/PLA composite. Appl Surf Sci, 2008, 255: 494-497

8 Wang X Y, Shan X Z, Wei M, et al. Preparation, mechanical properties and in vitro degradation of hydroxyapatite/poly-racemic lactic acid composite. J Chin Ceram Soc, 2007, 35: 1538-1540

9 Hu Q J, Cui W, Gong X H, et al. Study of mechanical properties of polylactide/hydroxyapatite composites prepared by in-situ polymerization. Chin Plast Ind, 2006, 34: 23-26

10 ISO-10993-5: 1999(E). International Organization for Standardization. Biological evaluation of medical devices-Part 5: Tests for in vitro cytotoxicity. 1999

11 ISO-10993-12: 1996(E). International Organization for Standardization. Biological evaluation of medical devices-Part 12: Sample preparation and reference materials. 1996

Open Access This article is distributed under the terms of the Creative Commons Attribution License which permits any use, distribution, and reproduction in any medium, provided the original author(s) and source are credited. 